\title{
Bunching-induced asymmetry in degenerate four-wave mixing with cold atoms
}

\author{
G. L. Gattobigio, ${ }^{1,2}$ F. Michaud, ${ }^{1}$ J. Javaloyes, ${ }^{3}$ J. W. R. Tabosa, ${ }^{4}$ and R. Kaiser ${ }^{1}$ \\ ${ }^{1}$ Institut Non Linéaire de Nice, UMR 6618 CNRS, 1361 route des Lucioles, F-06560 Valbonne, France \\ ${ }^{2}$ Dipartimento di Fisica dell'Universita' di Ferrara and INFN-Sezione di Ferrara, 44100 Ferrara, Italy \\ ${ }^{3}$ Department de Fisica Interdisciplinar, IMEDEA (CSIC-UIB), C/Miquel Marques, 21, E-07190 Esporles, Spain \\ ${ }^{4}$ Departamento de Fisica, Universidade Federal de Pernambuco, Av. Professor Luiz Freire, \\ $s / n$ Cidade Universitaria, 50670-901, Recife-PE, Brasil
}

(Received 1 June 2006; published 12 October 2006)

\begin{abstract}
We have investigated degenerate four-wave mixing in a sample of cold rubidium atoms. A red-blue asymmetry is observed for high intensities of the pumping beams. This asymmetry is explained by the spatial bunching of the atoms in the nodes or antinodes of the strong standing wave of the pump beams. This explanation is confirmed by different experimental configurations and by numerical simulations.
\end{abstract}

DOI: 10.1103/PhysRevA.74.043407

PACS number(s): 32.80.Pj, 42.50.Gy, 03.67.- a

Four-wave mixing (FWM) has been extensively studied in the past three decades and constitutes a very powerful spectroscopic technique both in frequency and time domain [1]. From a practical perspective, the possibility of performing wave-front reconstruction is one of the most fascinating applications of this nonlinear optical process. The use of cold atoms to obtain FWM optical phase conjugation benefits from the reduced atomic motion where broadening mechanisms such as transit time broadening and second-order Doppler effect can be completely eliminated. This allows us to obtain wave-front reconstruction over a large angular aperture between the pump and the probe beams [2,3]. In this regime one can also directly compare theoretical predictions with experiments without the need to perform any velocity averaging. For instance, the polarization dependence of the nondegenerate FWM spectrum has been measured in cold cesium atoms which is in complete agreement with the theoretical predictions [4]. Another interesting application associated with the FWM process is the possibility to investigate the atomic dynamics either in room temperature vapors [5] or in the domain of ultracold temperatures associated with laser cooled atoms. In particular, degenerate and nondegenerate FWM were employed to probe the quantized atomic motion of atoms in optical lattice [6,7]. The total FWM signal arises from the coherent superposition of the fields generated at different position along the nonlinear medium. Actually, for a spatially uniform atomic medium, this leads to the well-known phase matching condition which determines the propagation direction of the generated field in terms of the wave vectors of the incident waves. However, this situation is rather changed when the medium presents some spatial modulation where the generated field can pick up a wave-vector component associated with the Fourier decomposition of the spatial modulation of the medium, therefore affecting the overall efficiency of the nonlinear process and changing the propagation direction of the generated wave [8]. In this work we experimentally investigate the degenerate four-wave mixing (DFWM) process [9] in a sample of cold rubidium atoms both in the time and in the frequency domain using different polarization configurations for the DFWM beams. Note that we study DFWM where the frequency of both pump beams and the probe beam are identical, in contrast to many pump-probe spectroscopy experi- ments where the probe frequency has been scanned around the pump frequency. In most of the experiments using cold atoms, the pump beams have a fixed (red) detuning and the observed subnatural linewidths in nearly degenerate FWM have been explained by the dynamics of atoms in dissipative optical lattices [10]. We have registered the DFWM spectra for positive (blue) and negative (red) detuning of the pump and probe beams and observed an unexpected red-blue asymmetry for high intensity of the pumping beams. This asymmetry in the FWM spectrum can be explained by the presence of different spatial atomic distributions on the red and on the blue side of the atomic resonance. We associate this atom bunching to the dipole forces in strong fields with modulated intensity.

The experiment was performed in a sample of cold rubidium atoms obtained from magneto-optical trap (MOT). Our MOT scheme has been described elsewhere [11] and allows a time controlled switching of the trapping and repumping beams as well as the magnetic quadrupole field. The atom number can be adjusted in this experiment by a variable intensity of the repumping laser. Unless otherwise specified, the results shown in this paper have been obtained with a moderate number of atoms $\left(\sim 10^{9}\right)$ as more complex collective effects appear for the maximum number of atoms, of the order of $10^{10}$, we are able to trap. The DFWM beams are provided by the same laser (frequency $\omega_{L}$ ) and can be controlled in time to adjust the pulse duration. We employ the conventional backward DFWM configuration with two independent (not retroreflected) counterpropagating pumping beams (forward $F$ and backward $B$ ). Figure 1(a) shows a simplified scheme of the experimental configuration. The angle between the probe $(P)$ and forward beam $(F)$ has been maintained constant at around $0.2 \mathrm{rad}$. The waist of the laser beams $(w=6 \mathrm{~mm})$ is slightly larger than the size of our cloud. The polarization of the DFWM beams can be adjusted by appropriate wave plates and polarizing cubes placed along the beam paths. Table I summarizes the different combinations of polarization we have used for this work. In Fig. 1(b) we show the timing sequence of the experiment. First the atoms are collected and cooled for about $20 \mathrm{~ms}$, then the MOT trapping beams and the quadrupole magnetic field are switched off for about $1 \mathrm{~ms}$. During this time window, the 


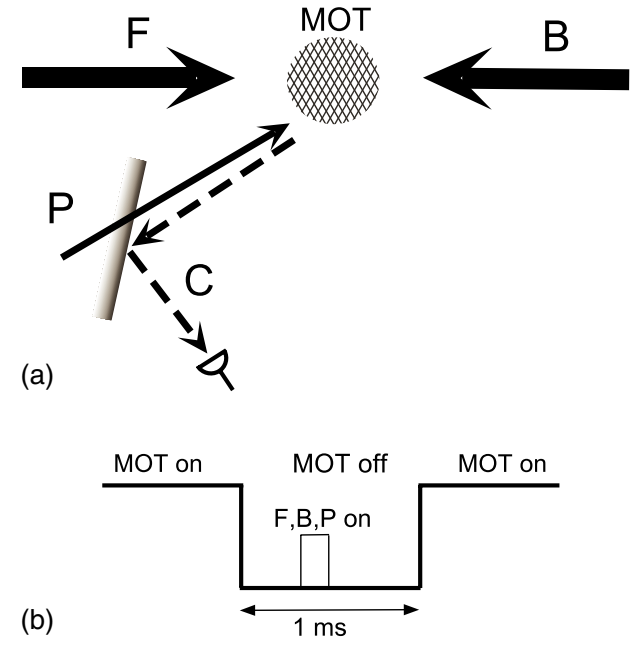

FIG. 1. (Color online) (a) Schematic setup of the experiment: two strong counterpropagating pump beams $(F$ and $B)$ and one weak probe beam $(P)$ yield the conjugate four-wave mixing signal $(C)$; (b) timing of the experiment, the MOT beams and magnetic fields are switched off during the short DFWM pulse.

DFWM beams are switched on and the generated phase conjugated beam, after being reflected off a beam splitter, is detected by a fast photomultiplier. The duration $\Delta T$ of the DFWM beam (in the range of 20 to $40 \mu \mathrm{s}$ ) has been adjusted to be short enough so that the strong pump beams do not influence the atom number at the turn off of each MOT sequence. The repumping laser of the MOT has been kept on during the DFWM sequence, in order to avoid losses of interacting atoms by optical hyperfine pumping. The DFWM spectra shown in this paper correspond to an integration over interaction times of several $\mu$ s typically after $20 \mu$ s of interaction. The precise value of the DFWM depends on the choice of these values, but the qualitative features discussed in this work are robust against a decent variation of these parameters.

We have first analyzed the case where the pump and probe beams have orthogonal linear polarizations $F \| B \perp P$, as this choice of polarization is known to yield large DFWM signals [4]. For this configuration, the signal originates from a coherence grating induced in the Zeeman sublevels by the orthogonal polarization components of the probe and pump fields. We record the DFWM spectrum as a function of the common frequency detuning $\delta=\omega_{L}-\omega_{a t}$ of DFWM beams in relation to the resonance frequency $5 S_{1 / 2}, F_{g}=3-5 P_{3 / 2}$, $F_{e}=4$ of the $D_{2}$ transition of rubidium ${ }^{85} \mathrm{Rb}$ (wavelength $\lambda=\frac{2 \pi}{k}=\frac{2 \pi c}{\omega_{a t}}=780 \mathrm{~nm}$, width of the resonance line

TABLE I. Various polarization configurations used in this paper.

\begin{tabular}{llll}
\hline \hline & $F$ & $B$ & $P$ \\
\hline$F\|B\| P$ & $\uparrow$ & $\uparrow$ & $\uparrow$ \\
$F \| B \perp P$ & $\uparrow$ & $\uparrow$ & $\leftrightarrow$ \\
$\sigma^{+}-\sigma^{+}$ & $\circlearrowright$ & $\circlearrowright$ & $\uparrow$ \\
$\sigma^{+}-\sigma^{-}$ & $\circlearrowright$ & $\circlearrowleft$ & $\uparrow$ \\
\hline \hline
\end{tabular}

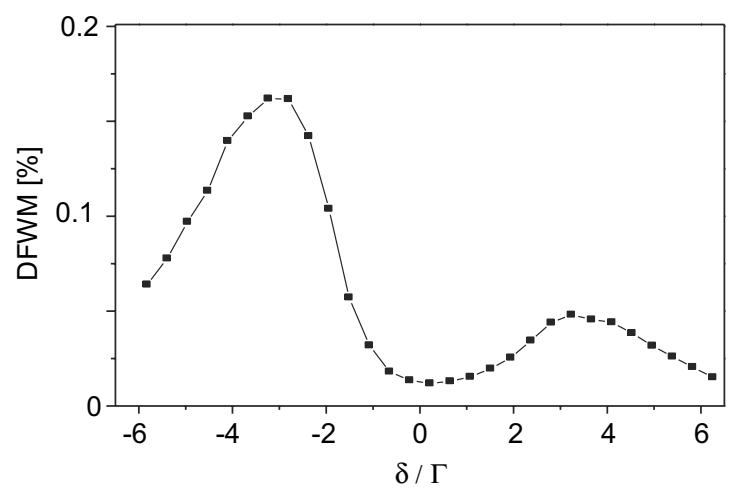

FIG. 2. DFWM spectrum for orthogonal polarizations $(F \| B \perp P)$ and strong saturation of the pump beams, $s=5$.

$\Gamma=5.89 \mathrm{MHz}$, saturation intensity $\left.I_{\mathrm{sat}}=1.6 \mathrm{~mW} / \mathrm{cm}^{2}\right)$. Assuming a uniform distribution of the atoms among the various Zeeman sublevels, we define the on-resonance saturation parameter $s=\frac{3}{7} \frac{I}{I_{\text {sat }}}$ by including an average over the ClebschGordan coefficients.

The spectrum obtained for pump intensities corresponding to a saturating parameter of about $s=5$ for the $F$ and $B$ pump beams and $s_{P}=0.5$ for the probe beam, is shown in Fig. 2 and consists of a double peak centered around the detuning $\delta$ $=0$. Note that the precise values (including the shape) of these DFWM spectra depend on the precise time window used for the evaluation, as illustrated by time resolved results shown below. The physical origin of this double peak structure for the $F \| B \perp P$ and $\sigma^{+}-\sigma^{-}$configurations lies in a combination of the saturation induced by the strong pump field and line center absorption due to the larger optical thickness around the resonance $[12,13]$. We have verified that with a reduced atoms number $\left(\sim 10^{8}\right)$ the line center absorption vanishes and there is no double peak structure for the $F\|B\| P$ and the $\sigma^{+}-\sigma^{+}$configuration for saturation values up to $s$ $=30$. As expected a double peak structure however remains for the $\sigma^{+}-\sigma^{-}$configuration [14]. Another possibility of double peak structure can be obtained for large probe beam intensities [15], but can be excluded with our choice of probe beam intensities. Note also that for DFWM in Doppler broadened hot atomic vapors, the average over the various velocity classes leads to a splitting even in the $F\|B\| P$ configuration. This can be understood by the fact that in the rest frame for each velocity class, the laser frequency of the pump beams are shifted by the Doppler effect and one has a situation which is no longer DFWM but nondegenerate FWM [14].

A more surprising feature however is the clear red-blue asymmetry: the DFWM signal is clearly larger for negative (red) detuning than for positive (blue) detuning. A model based on only the internal degrees of freedom of the individual atoms, taking into account all Zeeman sublevels of the relevant ground $\left(5 S_{1 / 2}, F_{g}=3\right)$ and excited $\left(5 P_{3 / 2}, F_{e}=4\right)$ states cannot predict such a red-blue asymmetry $[9,14,16]$. We neglect the influence of other hyperfine states in the excited level, separated by more than 20 linewidths, and the ground state, even further detuned, even though subtle polarization effects due to the neighboring excited state levels 
have been reported [17]. As our red-blue asymmetry has been robust against slight changes in alignment, intensity imbalance and atom number, we do not think that a change in the index of refraction of the effective medium [12] nor self-focusing [13] plays an important role in our experimental observation. We have however observed that for longer interaction times (up to $800 \mu \mathrm{s}$ ) the number of atoms after each MOT period of $20 \mathrm{~ms}$ (as monitored by the fluorescence signal at the end of the MOT period) is reduced for blue detuning of the pump beams. This loss of atoms is attributed to classical Doppler heating of atoms. For long enough interaction times (typically for DFWM pulses exceeding $100 \mu \mathrm{s}$ ), atoms can be accelerated beyond the velocity capture range of the MOT and cannot be recaptured by the next MOT period. This effect can also give rise to a more trivial red-blue asymmetry and must be ruled out when studying the bunching induced asymmetry.

As discussed below we attribute the asymmetry observed for short interaction times to the bunching of atoms along the standing wave pattern associated with the counterpropagating pumping beams. Dipole forces acting on atoms are known to change sign with the atom-laser detuning as the light shift induced by the laser depends on its detuning [18]. For large Rabi frequencies atoms are high field seekers at red detuning and low field seekers at blue detunings (as used, e.g., in atom mirrors [19]). The red-blue asymmetry in the DFWM signal presented in this paper appears when atoms are moving in a intense modulated standing wave with a light shift comparable to the kinetic energy of the atoms [20].

In order to investigate the origin of this asymmetry experimentally and theoretically, we have performed a number of control experiments which allow for clearer signatures corresponding to this interpretation and an easier comparison to theory. We have thus performed experiments with circular polarized pump beams. The pump beams $F$ and $B$ have either the same circular polarization $\left(\sigma^{+}-\sigma^{+}\right)$or opposite circular polarizations $\left(\sigma^{+}-\sigma^{-}\right)$. As the probe beam for the $\left(\sigma^{+}-\sigma^{-}\right)$ configuration has been kept linear (see Table I), the signal originates from a coherence grating induced in the Zeeman sublevels by the orthogonal polarization components of the probe and pump fields. Note that a minimal description for the $\left(\sigma^{+}-\sigma^{-}\right)$and the $F \perp B \| P$ configurations require a threelevel system, whereas the $F \| B \perp P$ is better described by a four-level system. On the other side, the $\left(\sigma^{+}-\sigma^{+}\right)$and the $F\|B\| P$ configuration are well described by two-level systems. A quantitative comparison between the various polarization configurations is hence not possible. The important point however is the change in the intensity modulation for these two configurations. In the $\sigma^{+}-\sigma^{+}$configuration, the polarization of the field is $\sigma^{+}$and constant in space but the amplitude of the local electric field is strongly modulated, and corresponding strong dipole forces will act onto the atoms. In the $\sigma^{+}-\sigma^{-}$configuration however, for identical intensities for the $F$ and $B$ beams, the local polarization is linear (with a spatially rotating axis) but the amplitude of this field is constant in space (neglecting at first order the influence of the probe beam). In this $\sigma^{+}-\sigma^{-}$situation the light shift of the atoms is not modulated in space and no dipole force is expected to occur. We thus predict a red-blue asymmetry for the $\sigma^{+}-\sigma^{+}$configuration but we do not expect any asymmetry for the $\sigma^{+}-\sigma^{-}$configuration.

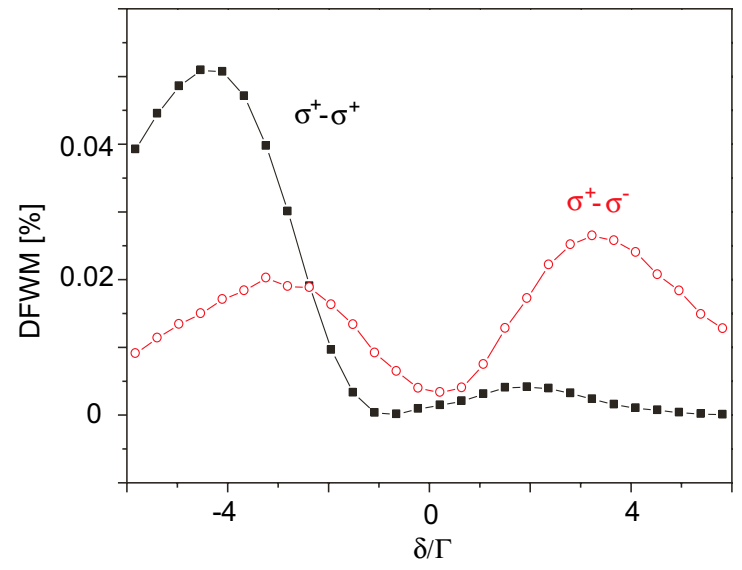

FIG. 3. (Color online) DFWM spectrum for circular polarizations of the pump beams, $\sigma^{+}-\sigma^{+}$(full squares) and $\sigma^{+}-\sigma^{-}$(open circles); pump beam saturation, $s=3.3$.

This is what we have observed in the experiment as shown in Fig. 3. We have recorded the DFWM spectrum for the two circular polarization configuration of the pumping beams in the regime of strong pump saturation $(s=3.3)$. As can be seen in Fig. 3, the red-blue asymmetry is strongly reduced for the case where the pump beams have opposite circular polarization and where no modulation of the light shift due to the pump beams is expected. This result strongly supports our explanation that mechanical effects based on dipole forces, absent in the $\sigma^{+}-\sigma^{-}$configuration, are at the origin of the observed red-blue asymmetry.

One should however bear in mind the mechanism for DFWM is different for these two polarization configurations. Indeed, in the $\sigma^{+}-\sigma^{-}$case, the strong pump beams $F$ and $B$ directly induce a coupling between different Zeeman sublevels, which are then probed by the weaker beam $P$. Comparing our $\sigma^{+}-\sigma^{-}$case, with linear probe polarization with a configuration using $F \perp B \| P$ would involve more closely related four-wave mixing mechanisms, as both configuration should be well described with a four-level model. One can thus compare the results of the $\sigma^{+}-\sigma^{-}$shown in Fig. 3 to those of Fig. 2. The important point is the change in the intensity modulation for these two configurations. In the $F \| B \perp P$ configuration, the polarization of the strong pump field is linear and constant in space but the amplitude of the local electric field is strongly modulated, with corresponding strong dipole forces acting on the atoms. However even such experiments would only allow for qualitative comparison, as optical pumping and the relative polarization of the probepump beams would not be the same. We have therefore chosen to use a comparison using a minimal change in the experiment (switching polarization of the $B$ beam from $\sigma^{+}$to $\sigma^{-}$). The main result is that we have systematically observed a strong red-blue asymmetry for strong enough pump beam for all polarization configurations, except for our $\sigma^{+}-\sigma^{-}$configuration, where we expect no spatial modulation of the light shift induced by the pump beams.

In order to further investigate the consequence of such mechanical effects on the DFWM signal, we have performed experiments, specifically designed for an easier comparison with theoretical simulations. Indeed, when the spatial distri- 


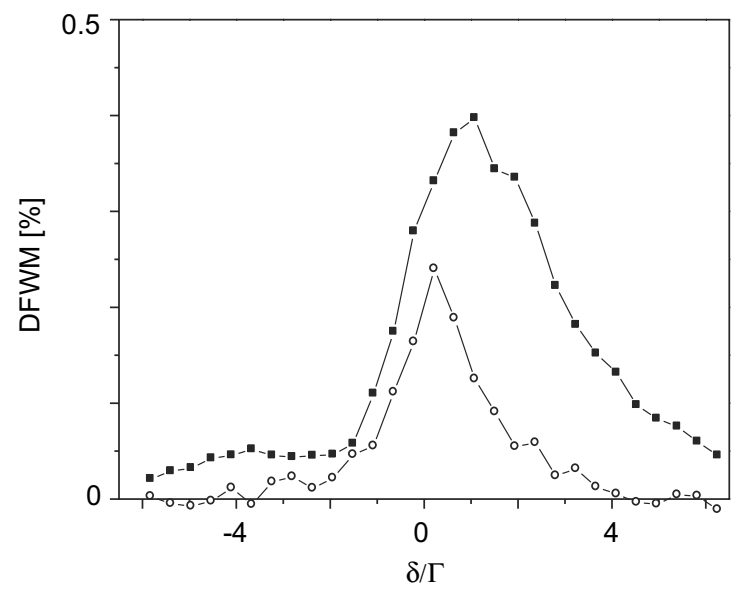

FIG. 4. DFWM spectrum for parallel polarizations of the pump and probe beams, $F\|B\| P$. Weak pump intensity $s=0.4$ (rescaled $\times 100$ ) (open circles) and strong pump intensity $s=4$ (full squares).

bution of the atoms is no longer uniform, one must take into consideration not only the coherences (Zeeman or optical coherences) but also the density modulation of the atoms. Let us, e.g., consider the situation with a strong spatial intensity modulation (e.g., $F\|B \perp P, F\| B \| P$ or $\sigma^{+}-\sigma^{+}$). As the intensity of the pump beam is increased up to the point when the induced light shifts are larger than the kinetic energy of the atoms, dipole forces are important enough to induce a bunching of the atoms. One now must evaluate whether bunching at the antinodes of the standing wave (for red detuning) will increase or decrease the DFWM signal in comparison with bunching at the nodes of the standing wave (for blue detuning). The theoretical description of DFWM including these effects of the external degrees of freedom of the atoms is somehow less complex if one considers all polarizations for both pump and probe field parallel, $F\|B\| P$. In contrast to the previous configurations, the signal now originates from an optical coherence grating of two-level atoms and Zeeman coherences in the ground state can be neglected. This allows for an easier numerical simulation of the experimental situation. In the low saturation limit, one thus expects an increased DFWM when atoms are bunched around the antinodes of the standing wave, increasing the average local intensity the atoms experience. This argument is supported by numerical simulations presented below. The experimental result are shown in Fig. 4.

In contrast to the orthogonal and circular configurations, where Zeeman coherences are responsible for the DFWM signal, no splitting is observed for the parallel configuration, as expected for two-level atoms at rest [9]. For low saturation of the pump beams, the DFWM is symmetrically centered around $\delta=0$ (Fig. 4, open circles). For larger saturation however, the maximum of the DFWM signal is shifted towards the blue $(\delta>0)$ side of the spectrum (Fig. 4, full squares). However this blueshift does not correspond to the asymmetry predicted above. By changing the saturation parameter of the probe beam and by carefully balancing the intensities of the forward and backward pump beams, we have verified in a series of experiments, that this blueshift is not caused by a residual light pressure of the pump and probe beam [21]. In fact, this blueshift for larger saturation parameter is due to the bleaching (saturation) of the nonlinearity of the atoms [8]. Indeed, for a two level atom, a perturbative description of the nonlinearity is only valid at low saturation of the atomic transition. For too large saturation, the nonlinearity will decrease again with a total bleaching of the transition for infinite saturation. When the saturation parameter of the pump beam exceeds a certain threshold, an increase of the intensity of the pump beams will lead to a decrease of the nonlinear DFWM signal. Correspondingly a bunching of atoms at the antinodes of the standing wave (high field seekers for red detuning) will not increase but decrease the DFWM in this regime. On the other hand, bunching of the atoms (by dipole forces) at the nodes of the standing wave (low field seekers for blue detuning) will lead to an increase of the DFWM. This is what we observe in our experiment. Note that for the experiments where a center line dip is present, the asymmetry is more striking at larger values of detuning, where this bleaching of the atomic response does not occur. This is how we explain the larger DFWM for red detuning in Figs. 2 and 3. A redshift of the DFWM around resonance would be expected for lower saturation parameters. However, the bunching of atoms due to the dipole forces of the pump field also depends on the saturation parameter and we cannot produce significant bunching in the low intensity limit where a redshift is expected. As detailed below we have confirmed these effects by numerical simulations using a two-level model for the atoms.

Before turning to the description of our numerical simulations, let us present further experimental results for the $F\|B\| P$ configuration. As we can register not only the DFWM signal at the end of the short pulse (all results presented above) but also the time resolved DFWM, the dynamics of DFWM can be probed. This is illustrated in Fig. 5.

As one can see in these figures, the DFWM is almost independent of time for low saturation. This is consistent with the explanation that for these values of the saturation parameter no bunching is obtained. Also the values for red and blue detuning are similar, as already shown in Fig. 4. For larger pump intensities however, one can see the red-blue asymmetry already shown in Fig. 4, but one can also clearly distinguish a strong time dependence (with initial oscillations) of the DFWM. This time dependence is another confirmation of the bunching induced DFWM as it is consistent with the time scales of the spatial motion of the atoms experiencing strong dipole forces in the modulated light field. This time-dependent DFWM could be exploited to measure the time-dependent bunching as explained below. Note that due to this strong time dependence for large saturation values, a more precise comparison between the various polarization configurations would require a time resolved comparison. This complexity will be illustrated in Fig. 6 for the results of our numerical simulations. Furthermore for large values of the saturation parameter there is not always a steady state, as one can lose atoms by Doppler heating. This makes a complete quantitative analysis almost impossible and we have thus extracted the results shown in Figs. 2-4 from a time window ranging from 10 to $40 \mu \mathrm{s}$. In order to further support our analysis of a bunching induced red-blue asymmetry, we have performed numerical simulations to es- 

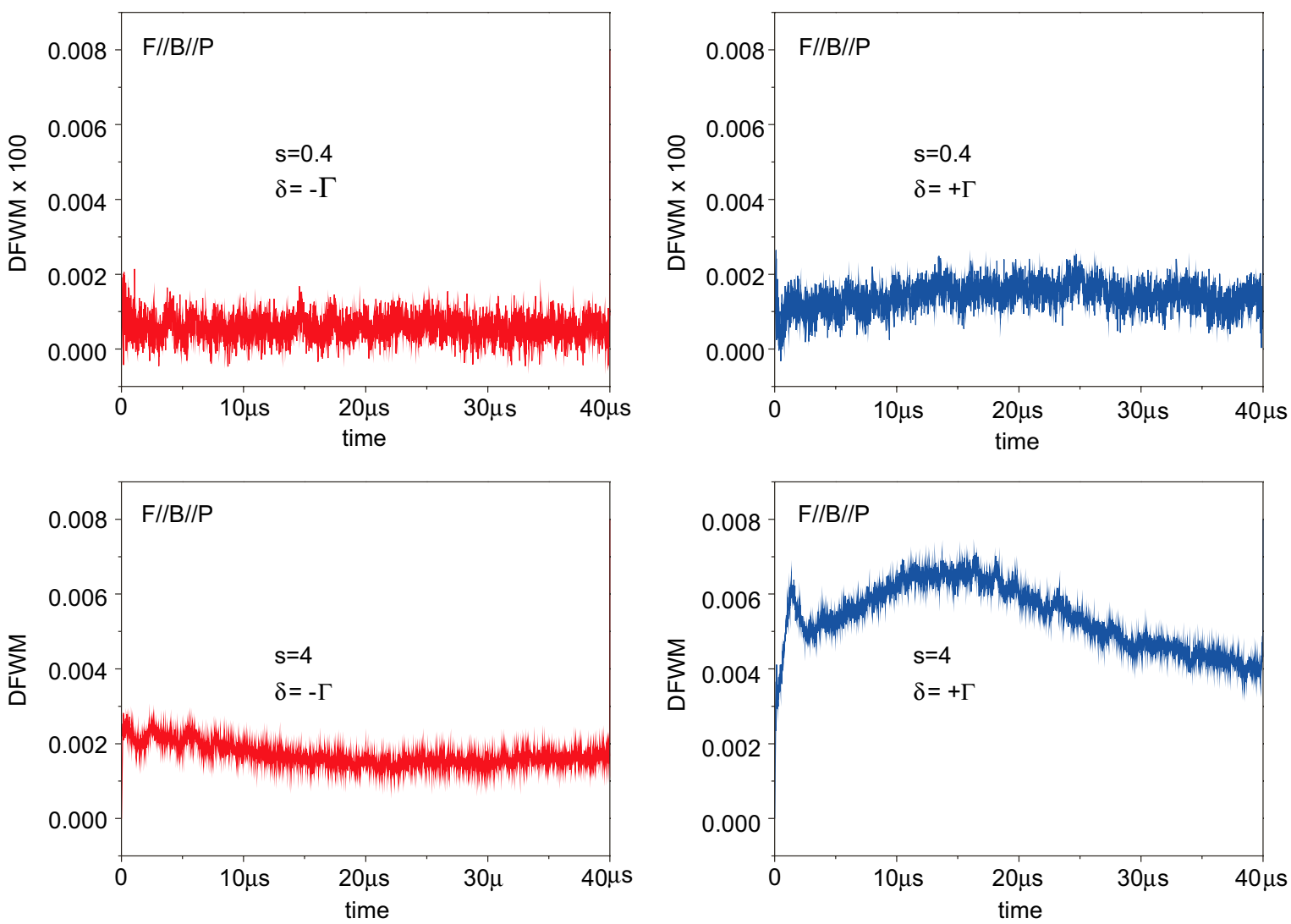

FIG. 5. (Color online) Time resolved DFWM signal for linear polarizations of the pump and probe beams, $F\|B\| P$. Upper curves, low saturation $s=0.4$ for red $(\delta=-\Gamma$, left curve) and blue $(\delta=\Gamma$, left curve) detuning. Lower curves, high saturation $s=4$ for red $(\delta=-\Gamma$, left curve) and blue ( $\delta=\Gamma$, left curve) detuning. Note the change of the vertical scale due to increased DFWM for larger saturation.

timate the spatial ordering induced by the strong standing light wave. Following the method described in Ref. [22], we used the dressed state basis and solved the coupled Heisenberg equations for the position, momentum, optical coherence, and populations for each atom. The reversible dynamics for each particle is interrupted by stochastic events, corresponding to stimulated emissions between dressed states and thus to a reset of the atomic internal degree of freedoms. In our numerical simulation, we have neglected the retroactions of the atoms onto the pump fields which could occur due to the stimulated or spontaneous radiation of the atoms. Such interaction can give rise to interesting col-

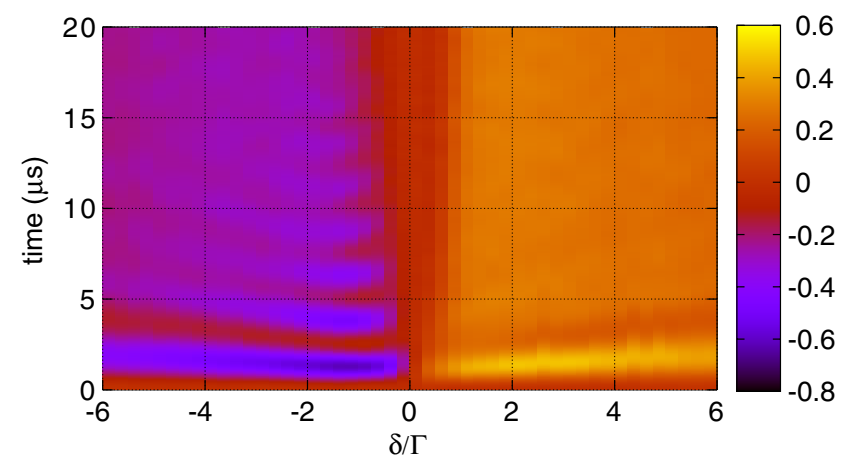

FIG. 6. (Color online) Numerical simulation of the bunching for a two level atom with a pump beam saturation of $s=4$. lective features [23] which are however not in the scope of this paper. Moreover, this approximation is justified by the relatively modest number of atoms used in the experiment. We expect the transition toward a collective regime to appear only above a critical value of atom number. Indeed, in the experiments we encountered more complex collective behavior for larger atomic clouds, when $N_{a t}>10^{10}$.

In order to characterize the atomic ensemble, we defined the bunching parameter within the cloud $b$ as the harmonic modulation of the atomic density distribution $\rho(z)$ at half the wavelength,

$$
b=\frac{k}{2 \pi} \int_{0}^{2 \pi / k} \rho(z) e^{2 i k z} d z
$$

As expected, the bunching parameter $b$ is an increasing function of the pump intensities. By an appropriate choice of the origin $z=0$ and of the relative phase between the two pump beams $F$ and $B$, we impose the standing wave to be proportional to $\sin (k z)$. Accordingly, due to the mirror symmetry $(z, F, B) \rightarrow(-z, B, F)$ of the problem, the bunching $b$ can be considered as a real valued quantity, up to finite size scaling fluctuations. The expected behavior consisting of bunching at the nodes (field minima) for blue detuning $(\delta>0)$ correspond to $b>0$, while bunching at the antinodes (field maxima) for red detuning $(\delta<0)$ correspond to $b<0$. In Fig. 6 we show the resulting time evolution of $b$ as a function of 
detuning for an initial temperature of the cloud of $T$ $=100 \mu \mathrm{K}$, a saturation parameter $s=4$ and an atom number $N=4096$. One can see that the bunching takes a nonnegligible value in a broad range of detuning values. However, it is vanishing in the vicinity of $\delta=0$ since in this case the dipole forces are too weak to sustain the periodic modulation against spontaneous emission recoils. Generalizing the calculations by Ref. [9], by explicitly including the density profile, the conjugate DFWM field is given in the limit of small optical thickness by the following expression:

$$
\kappa=4 s(1-2 i \delta) \int_{0}^{2 \pi / k} \frac{\rho(z) \sin ^{2} k z}{\left(1+4 \delta^{2}+4 s \sin ^{2} k z\right)^{2}} d z .
$$

The DFWM signal we measure is then given by $|\kappa|^{2}$. Assuming a harmonic ansatz for the spatial modulation of the atomic density $\rho(z)=(1+2 b)-4 b \sin ^{2} k z$, we can write the conjugate field $\kappa$ as

$$
\kappa=\kappa_{0}\left[\mathcal{I}_{1}(1+2 b)-4 b \mathcal{I}_{2}\right]
$$

with $\kappa_{0}=4 s(1-2 i \delta) /\left(1+4 \delta^{2}\right)$ and

$$
\mathcal{I}_{j}=\int_{0}^{2 \pi / k} \frac{\sin ^{2 j} k z}{\left(1+a^{2} \sin ^{2} k z\right)^{2}} d z .
$$

The two integrals $\mathcal{I}_{1}, \mathcal{I}_{2}$ can be evaluated numerically and only depend on the pump beam detuning $\delta$ and saturation $s$ through $a^{2}=4 s /\left(1+\delta^{2}\right)$. Assuming that the modulation amplitude is small, we can express the relative modification $r$ of the DFWM signal as

$$
r=\left|\frac{\kappa(b)}{\kappa(0)}\right|^{2}-1 \approx 4 \alpha b+O\left(b^{2}\right)
$$

with $\alpha=\left(1-2 \mathcal{I}_{2} / \mathcal{I}_{1}\right)$. At this point, one can notice that $|\kappa|^{2}$ and $\alpha$ depends on $\delta$ only through $\delta^{2}$. Thus the absolute DFWM signal $|\kappa|^{2}$ and its bunching induced modification $r$ would be symmetric. It can be seen from the expression of $r$ that this symmetry is broken only by the bunching since it is an antisymmetric function of the detuning, i.e., $b<0$ (respectively, $>0$ ) if $\delta<0$ (respectively, $>0$ ). It is possible to invert this problem and to evaluate the spatial modulation from the modified FWM signal $r$. Using this approach one could estimate the bunching from experimental time resolved data. However one must consider that $\alpha$ is negative for $a \leq 2.2$ and positive above this value. This means that either for very small saturation or very large detuning, the DFWM signal is enhanced on the red side and diminished on the blue side. This is not however the case that we considered here and we are in a regime where the Rabi frequency in the high intensity region of the standing wave is sufficiently large not only to saturate the nonlinearity but also to practically cancel its magnitude.

We can now compare the numerical results to our experimental data. First we confirm that for the parallel polarization $F\|B\| P$, there is no splitting in the DFWM spectrum. Also we find that for the larger saturation parameters $s=4$ of our experiment, the DFWM is larger for positive (blue) detuning. Figure 7 illustrates this asymmetry for detuning of $\delta= \pm 0.5 \Gamma$. Transient bunching of several percent are pre-
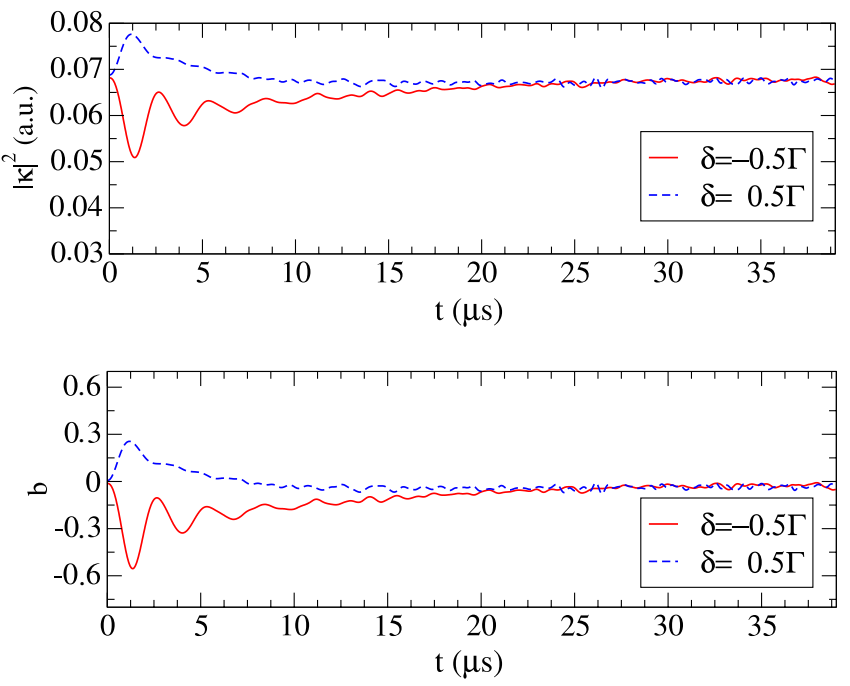

FIG. 7. (Color online) Numerical simulation for the time resolved DFWM signal (upper curve) and bunching (lower curve) for $\delta= \pm 0.5 \Gamma$ and $s=4$.

dicted and the time dependence of the bunching resembles the DFWM of Fig. 5. For saturation parameter of $s=0.4$ we do not obtain noticeable bunching, and the corresponding DFWM signal is again symmetric around $\delta=0$. We note that the region of the inverted red-blue asymmetry DFWM is larger in the experiment than in the simulation. Several effects might explain this difference, such as an oversimplified two-level system or an inhomogeneous intensity distribution of the DFWM beams. The purpose of this paper is to demonstrate the importance of the spatial rearrangement of the atoms for a DFWM signal, but we did not aim at a quantitative description of our signals.

In conclusion, we reported observation of DFWM with cold atoms, both for red and blue detuned light. We observe a red-blue asymmetry when atoms are exposed to dipole forces in the standing wave of the strong pump beams. The origin for an asymmetry of FWM spectra have not been reported so far and must be considered in particular when cold atoms are used as the nonlinear medium. We expect that it will be possible to increase by a considerable amount the efficiency of FWM in our cloud of cold atoms and it might then be interesting to study nonclassical features (squeezing) of the FWM signal and also add a cavity around the atomic cloud to yield self-oscillation [13,24]. Other possibilities with our pump-probe geometry include the combination of gain provided by the pump beams and multiple scattering if the optical thickness of the cloud is large. This could lead to the realization of a random laser [25] using cold atoms as a scattering and gain medium.

The authors thank G. Labeyrie for valuable help on the experimental setup. The authors acknowledge the financial support of CNRS and of the PACA Region. One of the authors (J.W.R.T.) would like to thank CNRS, CAPESCOFECUB, and CNPq for travel support. One of the authors (J.J.) acknowledges support from the program Juan de la Cierva through Grant No. TIC2002-04255-C04-03. 
[1] M. D. Lukin, P. R. Hemmer, and M. O. Scully, Adv. At., Mol., Opt. Phys. 42B, 347 (2000).

[2] L. Hilico, P. Verkerk, and G. Grynberg, C. R. Acad. Sci. III 315, 285 (1992).

[3] J. W. R. Tabosa, S. S. Vianna, and F. A. M. de Oliveira, Phys. Rev. A 55, 2968 (1997).

[4] A. Lezama, G. C. Cardoso, and J. W. R. Tabosa, Phys. Rev. A 63, 013805 (2001).

[5] D. S. Glassner and R. J. Knize, Phys. Rev. Lett. 74, 2212 (1995).

[6] B. Lounis, P. Verkerk, J. Y. Courtois, C. Salomon, and G. Grynberg, Europhys. Lett. 21, 13 (1993).

[7] A. Hemmerich, M. Weidermuller, and T. Hänsch, Europhys. Lett. 27, 247 (1994).

[8] M. Yocota and P. Yeh, J. Opt. Soc. Am. A 15, 2678 (1998).

[9] R. L. Abrams and R. C. Lind, Opt. Lett. 2, 94 (1978); 3, 205 (1978).

[10] G. Grynberg and C. Robillard, Phys. Rep. 355, 335 (2001).

[11] G. Labeyrie, F. de Tomasi, J. C. Bernard, C. A. Müller, C. Miniatura, and R. Kaiser, Phys. Rev. Lett. 83, 5266 (1999).

[12] G. Birkl, M. Gatzke, I. H. Deutsch, S. L. Rolston, and W. D. Phillips, Phys. Rev. Lett. 75, 2823 (1995).

[13] M. Oria et al., Opt. Lett. 14, 1082 (1989).

[14] M. Pinard, P. Verkerk, and G. Grynberg, Phys. Rev. A 35, 4679 (1987).

[15] R. T. Bratfalean, G. M. Lloyd, and P. Ewart, J. Opt. Soc. Am.
B 16, 952 (1999).

[16] R. W. Boyd, M. G. Raymer, P. Narum, and D. J. Harter, Phys. Rev. A 24, 411 (1981).

[17] S. Balik, R. Olave, C. I. Sukenik, M. D. Havey, V. B. Datsyuk, I. M. Sokolov, and D. V. Kupriyanov, Phys. Rev. A 72, 051402(R) (2005).

[18] C. Salomon, J. Dalibard, A. Aspect, H. Metcalf, and C. CohenTannoudji, Phys. Rev. Lett. 59, 1659 (1987).

[19] V. I. Balykin, V. S. Letokhov, Yu. B. Ovchinnikov, and A. I. Sidorov, Phys. Rev. Lett. 60, 2137 (1988).

[20] M. Schiffer, E. Cruse, and W. Lange, Opt. Commun. 116, 143 (1995).

[21] S. Guibal, C. Mennerat-Robilliard, D. Larousserie, C. Triche, J.-Y. Courtois, and G. Grynberg, Phys. Rev. Lett. 78, 4709 (1997).

[22] J. Chen, J. G. Story, and R. G. Hulet, Phys. Rev. A 47, 2128 (1993).

[23] C. von Cube, S. Slama, D. Kruse, C. Zimmermann, P. W. Courteille, G. R. M. Robb, N. Piovella, and R. Bonifacio, Phys. Rev. Lett. 93, 083601 (2004); J. Javaloyes, M. Perrin, G. L. Lippi, and A. Politi, Phys. Rev. A 70, 023405 (2004).

[24] L. Hilico, C. Fabre, and E. Giacobino, Europhys. Lett. 18, 685 (1992).

[25] N. M. Lawandy, R. M. Balachandran, A. S. L. Gomes, and E. Sauvain, Nature (London) 369, 436 (1994). 